\title{
Observing of polar cap dynamic by Greenland chain of magnetic stations
}

\author{
Barkhatov N.A. ${ }^{1}$, Revunov S.E. ${ }^{2}$, Smirnova Zhanna. ${ }^{3}$, Cherney O.T $^{4}$ \\ ${ }^{1}$ Minin Nizhny Novgorod State Pedagogical University (Minin University), Nizhny Novgorod, Russian \\ Federation,nbarkhatov@inbox.ru \\ ${ }^{2}$ Minin Nizhny Novgorod State Pedagogical University (Minin University), Nizhny Novgorod, Russian \\ Federation, revunov@inbox.ru \\ ${ }^{3}$ Minin Nizhny Novgorod State Pedagogical University, Nizhny Novgorod, Russian Federation \\ z.v.smirnova@mininuniver.ru \\ ${ }^{4}$ Minin Nizhny Novgorod State Pedagogical University, Nizhny Novgorod, Russian Federation, \\ fiolet1975@mail.ru
}

\begin{abstract}
Observing technique of location high latitude zones of geomagnetic activity: polar cap, auroral oval and subauroral zone is demonstrated by the data from Greenland chain of magnetic observatories. Thebelonging of a particular observatory to the one of the indicated three zones is determined on the basis of self-training artificial neural network of Kohonen layer type. Amplitude-frequency specters of the geomagnetic data analysis during some quiet and disturbed days in 1998 serve as input data for calculation. To estimate the accuracy of our classification of zones of geomagnetic activity a special technique was designed - the results of classification were compared with a model of the boundary of the polar aurora oval, which is based on the analysis of satellite data from space crafts DMSP on distribution of particles. The correspondence of classification outcomes to this model is up to $60 \%$. The outcomes of our research testify to the possibility of the using of the data from the Greenland chain of magnetic stations to control the temporary dynamics of location of the three areas of high latitude space in real time.
\end{abstract}

Key words: Magnetic recording, magnetic observatory, geomagnetic storm, magnetosphere, artificial neural network, solar activity, forecast.

\section{INTRODUCTION}

The Greenland chain of magnetic stations register geomagnetic field temporary behavior in space points, which are simultaneously located in a polar cap, in auroral zone and in subauroral zone in periods of magnetospheric disturbances. Is it possible using the data of the Greenland chain to control dynamics of spatial position of the above given important areas of high latitude space in real time? To answer this question, in the given work, the technique of classification of amplitude-frequency spectra for geomagnetic disturbances registered by stations of the Greenland chain is created. The classification of spectra with the help of self-learning artificial neural network (ANN) of layer Kohonen type (fig. 1) was applied with the purpose of determination of spatial position of the given characteristic polar zones. This technique was applied for numeric data from the Greenland stations during particular magnito-quiet and magnito-disturbed days in 1998.

However, the neural network can successfully classify only in the case when the available data set (in our case - spectra of studied disturbances) does not contradict the offered design of division into classes. Division into classes - polar cap, oval of polar auroras, subauroral zone - testifies to the presence of particular features in the spectra for each of the high latitude areas. Then, the obtained three classes are physically justified. This can be shown by searching the features in spectra of the polar area for day and night time sectors, and also by the correlation analysis for the high latitude spectra of the magnetic field and IMP. Really, recently the attention has been paid to the outcomes of the combined spectral analysis of the Earth magnetic field and parameters of interplanetary plasma [1].

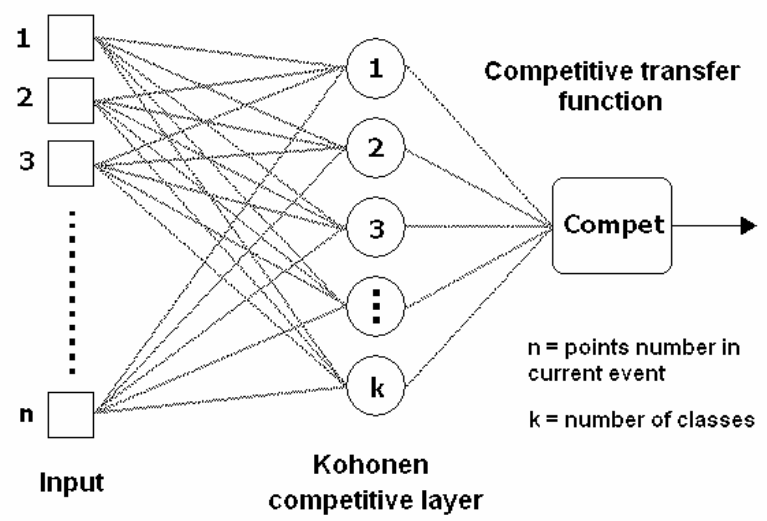

Figure 1:The architecture of Kohonen Self-Organizing Map 
Barkhatov N.A et al., International Journal of Emerging Trends in Engineering Research, 8(5), May 2020, 1552 - 1555

\section{DATA AND RESEARCH TECHNIQUE}

During the research, the data covering 1998 during magneto-quiet (Q-days) and magneto-disturbed (D-days) days were studied. The selection of quiet and disturbed days was conducted on the basis of Kp index observations. The Greenland map is shown in the geomagnetic coordinates with stations highlighted. In the first series of numerical experiments for each hour of selected day, classification of amplitude-frequency spectra of geomagnetic disturbances was executed for the stations of the chain. The calculation of spectra was conducted with the help of fast Fourier transform procedure, which presented a signal from each station as a numerical array for neural network entry.

The spectra by the data of three components of a magnetic field and its module were classified into three classes. In outcome for each selected day, the neural network gave 4 groups of answers. Each group contained 24 sets of stations (for each hour), subdivided into three zones. For convenience of classification outcome interpretation, each group of neural network answers was presented graphically.

Each segment in a figure corresponds to certain station (see a fig. 2). The color shows three selected classes. Ideally, the black segments - stations correspond to the middle latitude zone (class 1), dark gray segments - to the oval of polar auroras (aurora zone, class 2) and bright gray segments - to the polar cap (polar zone, class 3). The error of classification sometimes leads to the mixture of these areas. Nevertheless, the classification of station chain

spectra into three classes runs successfully.

The classification assessment was carried out on the basis of DMSP satellite data on particle emergence. They reflect approximate boundaries of the polar auroras oval (//sd-www.jhuapl.edu/aurora/) on the basis of particle emergence model in the polar area by b2i index calculation. For each researched day, 24 simulated images of aurora zone (one over each hour) were chosen. The model image of polar aurora zone boundary is obtained by the cutting of segments from the general image for each hour.

\section{RESULTS OF NUMERICAL EXPERIMENTS}

The comparison of outcomes obtained by the classification method with the model oval demonstrates some uncertainty at the determination of polar zones. In this connection a step-by-step algorithm is suggested below to clear up the designation of classes. For this purpose additional classification of the researched data into two classes was done. The described algorithm is applied below for outcomes of the fast Fourier transformation. The result of processing for two days is presented in fig. 1.

1. Classification of spectra into three classes.

2. Classification of spectra into two classes.

3. Superposition of the outcomes of classification.

4. Designation of names to zones in poorly reliable segments.

5. Designation of names to solitary, «falling out» fragments in segments.

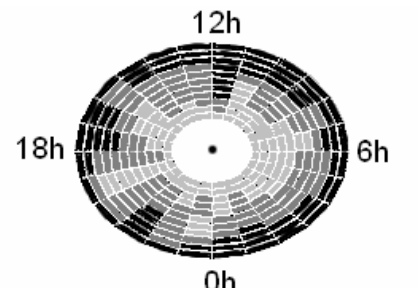

processing neural network answer (24.09.1998)
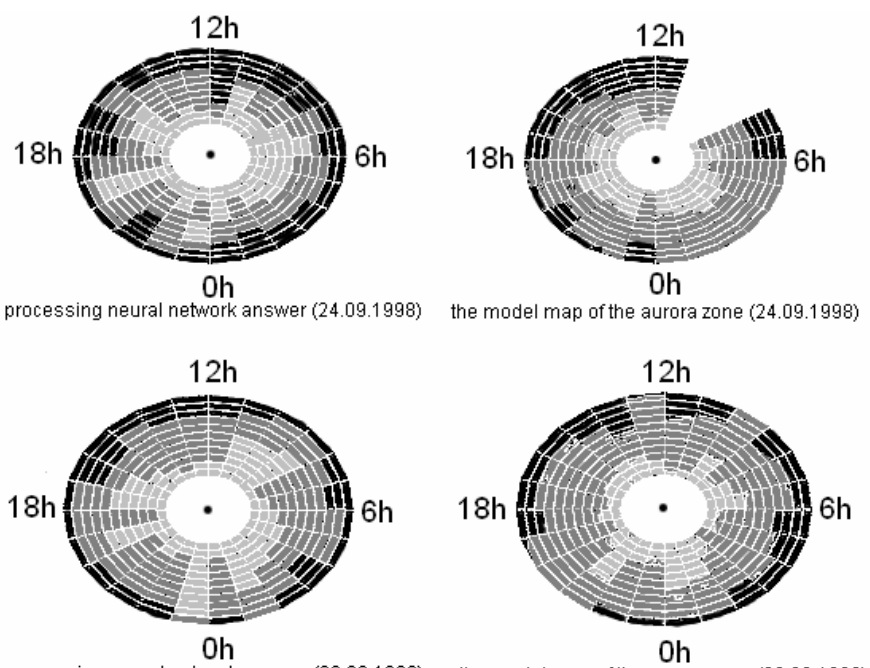

processing neural network answer (26.08.1998)

the model map of the aurora zone (26.08.1998)

Figure 2: An overall picture of the network answers after processing (left) and model construction of the oval by the data on energy of electrons (right) participating in it

The objective estimation of coordination level of algorithmically treated classification and model approaches was conducted by special algorithm. In the final picture of network outputs (after processing) and in the picture with model oval design, three series of values were separately calculated: the number of fragments of middle latitude segments, the number of fragments of oval segments and the number of cap segments for each hour. A factor of similarity was further calculated:

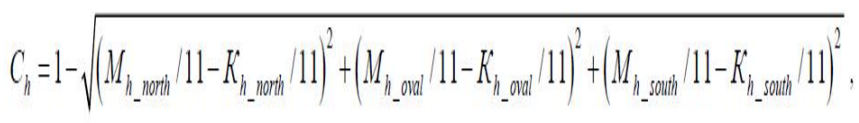

where Mhand $K h$ - fragment number of model array segments and neural network output-array, accordingly. Number $h$ of a segment means LT. The number 11 in denominator of each term in the formula corresponds to number of magnetic stations. Thus, the division of segment fragment number by the number of stations yields theweight of each part (answering to particular zone) of the segment. The calculation of the similarity factor takes into account the area of the compared parts of segments and their relative arrangement. The proximity of the coefficient to 1 testifies about the best coordination of the classification and model data. The mean concurrence of our outcomes with a model oval for perturbed days was $61-65 \%$.

\section{COMPARISON OF ALGORITHMIC CLASSIFICATION AND EMPIRICAL MODELS}

The designed technique and the created ANN as a tool of its implementation allow to conduct an estimation of the position of the southern boundary of the oval of polar auroras once per hour round the clock. We have compared the obtained outcomes with calculations on the basis of analytical ratios, 
which describe the position of this oval's boundary on the basis of correlation equations. These equations are obtained by the Polar geophysical institute (PGI) researches on the basis of comparison of the satellite data (satellites of DMSP series) with indices of the geomagnetic activity AL and Dst $[3,12]$. In fig. 3, as an example of such comparison, the temporary dynamics of the oval's southern boundary latitude according to our calculations, according to the satellite data, and according to calculations by formulas from $[4,11]$ is shown. One can see, that the position of the oval's southern boundary defined with the help of the magnetic data of the Greenland chain of stations can be used alongside with other modern methods for control, especially during the absence of satellite observations. It is worth noting that it is possible to organize such control in real time.

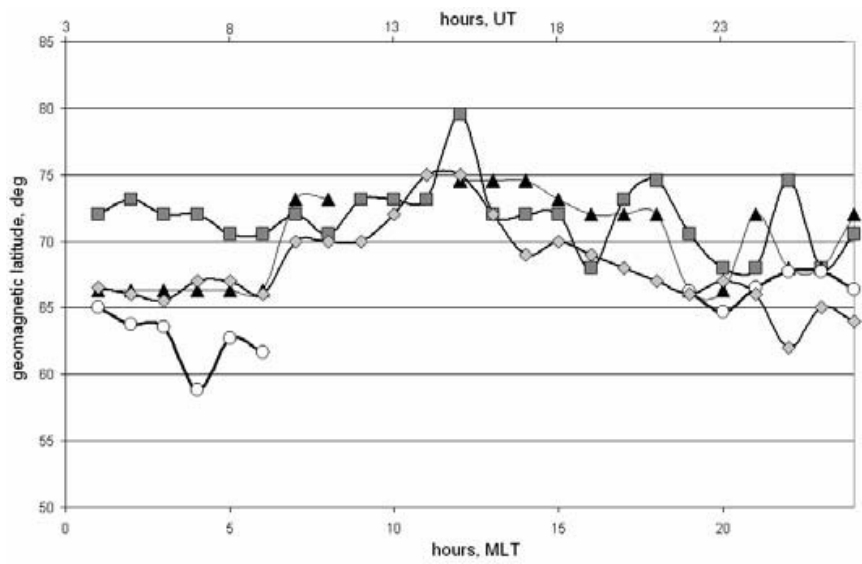

Figure 3: Determination of the auroraloval's southern boundary position on the basis of satellite observations (triangles), according to the data of the Greenland chain of magnetic stations (squares), by the correlation equations (diamonds), and position of the border in the night sector (circles) on magneto-disturbed day 24.09.98

\section{CONCLUSION}

To look for spectral characteristics peculiarities in day and night time auroral oval sectors, the magneto-disturbed day September 24, 1998 was selected since in these intervals, according to [7], different types of oscillations can be observed in spectra. This day at 23.20 UT, as it is noted in [8], the interplanetary shock wave reached the patrol space vehicle Wind, and at 23.44 UT reached the Earth magnetosphere and caused substorm expansion phase. For simplification of comparison of spectra and search of features within them, spectra of three stations - Thule (THL), Umanaq (UMQ) and Narsarsuaq (NAQ) are simultaneously and reciprocally considered. The first station precisely belongs to the area of the polar cap, and others can be in the oval auroral zone or in the subauroral zone.
Table 1: Average values of correlation coefficient at the component-wise analysis of spectral curves of ground stations with space vehicle Wind curves.

\begin{tabular}{|c|c|c|c|c|c|c|c|c|}
\hline $\begin{array}{l}\text { Components } \\
\text { of the } \\
\text { geomagnetic } \\
\text { field }\end{array}$ & IMF & $R_{a r}$ & $\begin{array}{l}\text { Components } \\
\text { of the } \\
\text { geomagnetic } \\
\text { field }\end{array}$ & IMF & $\mathrm{R}_{\mathrm{w}}$ & $\begin{array}{c}\text { Components } \\
\text { of the } \\
\text { geomagnetic } \\
\text { field }\end{array}$ & IMF & $\mathbf{R}_{a v}$ \\
\hline THL E & $\mathrm{Bx}$ & \multirow{3}{*}{0,96} & UMQ E & $\mathrm{Bx}$ & \multirow{3}{*}{0,85} & NAQE & $\mathrm{Bx}$ & \multirow{3}{*}{0,48} \\
\hline THL H & $\mathrm{Bx}$ & & UMQ H & $\mathrm{Bx}$ & & $\mathrm{NAQH}$ & $\mathrm{Bx}$ & \\
\hline THL Z & $\mathrm{Bx}$ & & UMQ Z & $\mathrm{Bx}$ & & $\mathrm{NAQZ}$ & $\mathrm{Bx}$ & \\
\hline THL E & By & \multirow{3}{*}{0,96} & UMQE & By & \multirow{3}{*}{0,86} & NAQE & By & \multirow{3}{*}{0,55} \\
\hline THL H & By & & UMQ H & By & & $\mathrm{NAQH}$ & By & \\
\hline THL Z & By & & UMQ Z & By & & $\mathrm{NAQZ}$ & By & \\
\hline THL E & $\mathrm{Bz}$ & \multirow{3}{*}{0,93} & UMQ E & $\mathrm{Bz}$ & \multirow{3}{*}{0,91} & NAQE & $\mathrm{Bz}$ & \multirow{3}{*}{0,66} \\
\hline THL H & $\mathrm{Bz}$ & & UMQ H & $\mathrm{Bz}$ & & $\mathrm{NAQ} \mathrm{H}$ & $\mathrm{Bz}$ & \\
\hline THL Z & $\mathrm{Bz}$ & & UMQ Z & $\mathrm{Bz}$ & & NAQ Z & $\mathrm{Bz}$ & \\
\hline
\end{tabular}

The detailed comparison of spectra of the geomagnetic field at these stations and IMP disturbances shows, that the highest correlation between them is observed for the station, which is classified by ANN as a polar cap. When moving to subauroral zone, the correlation between spectra of geomagnetic field and IMP components gradually decreases. Such outcome is expected, since the space vehicle Wind measures parameters of magnetic field falling exactly in the polar cap. These outcomes are presented in Table 1. Here, the average numbers of correlation coefficients are submitted by component-wise comparison for the day time interval 11-12 UT.

Search of characteristic peculiarities in oscillations at the station referred by ANN to the auroral oval showed, that in the midnight sector for all components of geomagnetic field, the peak for frequency of $7-8 * 10^{-3} \mathrm{~Hz}$ is present (less evident within the day time). According to $[5,10]$, it corresponds to the presence of oscillations Pc4. For example in fig. 4, the spectrum for the polar cap is shown by dotted line, for the subauroral zone, which has a peak on frequency of $7,5 * 10^{-3}$ $\mathrm{Hz}$ - by solid line. In the polar cap no such peak is observed.

The analysis of the spectrum adequate by results of ANN of classification to the subauroral zone station testifies to the presence of a characteristic peak for frequencies $0,7-1,1 * 10^{-3}$ $\mathrm{Hz}$ (fig. 5). The spectrum for a polar cap is plotted by a dotted line, for subauroral zone - by a solid line. The given low-frequency peak can be caused by the own oscillations of the closed force lines of the Earth magnetic field [6,9]. It is important, that in the polar cap and subauroral oval spectra, the given peak is not observed. 


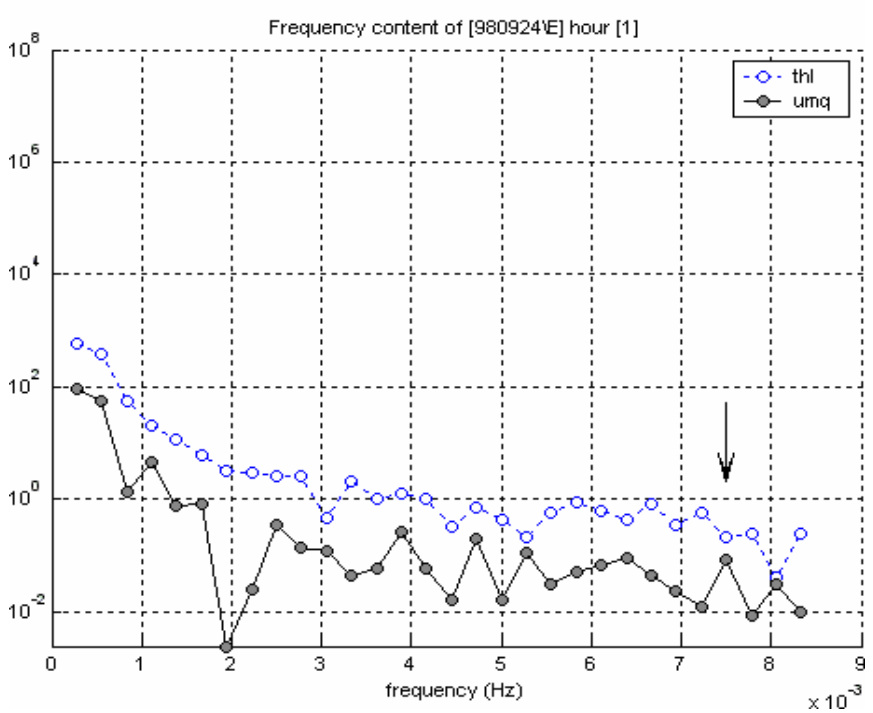

Figure 4: Comparison of the geomagnetic field E-component spectra from two stations Thule (THL, polar cap) and Umanaq (UMQ, auroral a zone) for night (00-01 UT) interval

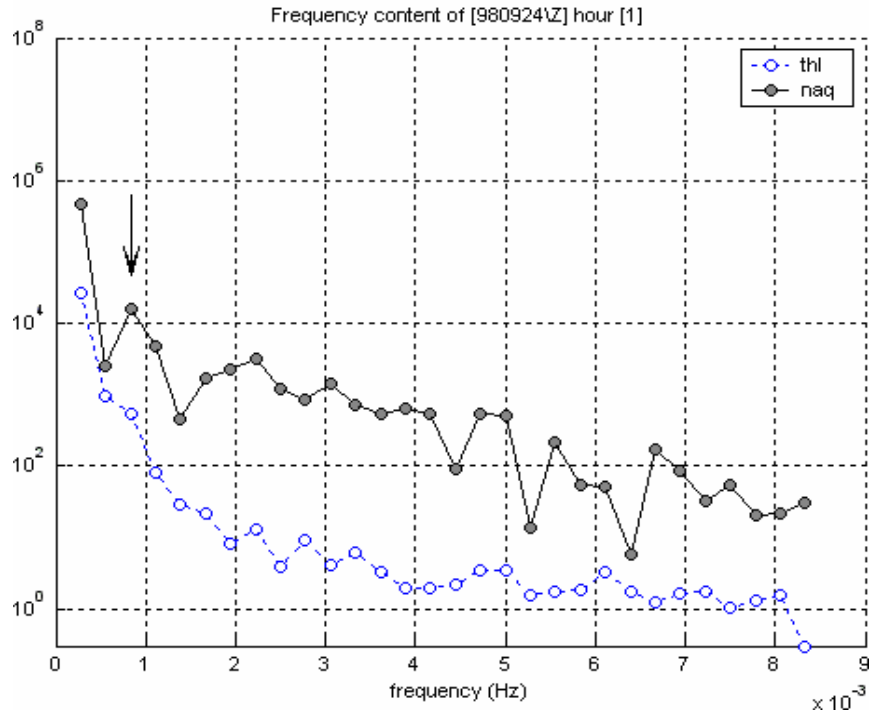

Figure 5:Comparison of the geomagnetic field E-component spectra from two stations Thule(THL, polarcap) and Umanaq (UMQ, auroralazone)fornight (00-01UT)interval

\section{ACKNOWLEDGEMENT}

This work was supported by RFBR grant No. 18-35-00430 and the State order of the Ministry of Education and Science of the Russian Federation No. 5.5898.2017 / 8.9.

\section{REFERENCES}

1. Vorobjev V.G., Yagodkina O.I. Effect of Magnetic Activity on the Global Distribution of Auroral Precipitation Zones // Geomagnetism and Aeronomy, V. 45. 2005. P. 438

2. Zaytcev A.N., Ivanov V.V. Time-frequency characteristics of geomagnetic disturbances with the periods of 1-30 mines. Geomagnetism and Aeronomy, T. 35. 1995. P. 165
3. ManakovaYu.V., Pekhteleva K.A., Barkhatov N.A., Revunov S.E. Space-time analysis of perturbations of the Pc4-5 range during periods of magnetic storms by the correlation-skeleton method // Vestnikof Minin University. 2016. no. 1. pp. 1-6.

4. Barkhatov N.A. Development of methods for predicting the geomagnetic state of the magnetosphere based on the search for fundamental laws of solar-terrestrial relationships // Vestnikof Minin University. 2013. no. 2. pp. $1-11$.

5. Barkhatov N.A., Revunov S.E., Vinogradov A.B. The classification algorithm for MHD wavelet-skeleton spectral patterns of geoeffective plasma flows in the solar wind // Vestnikof Minin University. 2014. no. 3. pp. 1-6.

6. Barkhatov N.A., V.G. Vorobjev, S.E. Revunov, O.M. Barkhatova, and O.I. Yagodkina Substorm Activity and Orientation of the Front of a Shock Wave of an Interplanetary Magnetic Cloud // Geomagnetism and Aeronomy, 2019, Vol. 59, No. 4, pp. 398-406. (C) Pleiades Publishing, Ltd., 2019.

https://doi.org/10.1134/S0016793219040042

7. Barkhatov N.A., Revunova E.A., Barkhatova O.M., Romanov R.V., Revunov S.E. Studying the relationship of localization parameters of solar sources of magnetic clouds with their characteristics and substorm activity // Solar-Terrestrial Physics. 2019. Vol. 5. Iss. 3. P. 59-67. DOI: 10.12737/stp-53201907.

8. Barkhatov N.A., Revunov S.E., Mukhina M.V., Gruzdeva M.L., Cherney O.T.and Smirnova Z.V. Establishing the orientation of shock wave plane of solar wind magnetic cloud for conclusions about the level of auroral substorm activity. JP Journal of Heat and Mass Transfer. Volume 17, Issue 1, Pages $195 \quad$ - $202 \quad$ (June 2019) http://dx.doi.org/10.17654/HM017010195, 2019

9. Barkhatov, N.A., Revunov, S.E., Vorobjev, V.G., and Yagodkina, O.I., Studying the relationship between high-latitude geomagnetic activity and parameters of interplanetary magnetic clouds with the use of artificial neural networks// Geomagn. Aeron., 2018, vol. 58, no. 2, pp. 147-153.https://doi.org/10.7868/S0016794018020013

10. Barkhatov N.A. , Revunov S.E., Smirnova Zh.V., Cherney O.T. and Mukhina M.V. Recovery of gaps in records of Geomagnetic observatories by Neural Network // International Journal of Emerging Trends in Engineering Research (IJETER) Volume 8 No. 4 (2020) p. 1243 - 1246 DOI: https://doi.org/10.30534/ijeter/2020/48842020

11. Barkhatov, N.A., Dolgova, D.S., and Revunova, E.A., Dependence of the geomagnetic activity on the structure of magnetic clouds // Geomagn. Aeron.,2019, vol. 59, no. 1, pp. 16-26.

https://doi.org/10.1134/S001679321901002X

12. Lauretis M. De, P. Francia, M. Vellante, A. Piancatelli, U. Villante, D. Di Memmo ULF geomagnetic pulsations in the southern polar cap: Simultaneous measurements near the cusp and the geomagnetic pole// J. of Geophys. Res., Vol. 110, No A11204, 2005. doi:10.1029/2005JA011058

13. Xiaoyan Zhou, Bruce T. Tsurutani Interplanetary shock triggering of night side geomagnetic activity: Substorms pseudo breakups, and quiescent events// J. of Geophys. Res., Vol. 106. No A9. 2001. P. 18957-18967 